\title{
Pattern and factors associated with congenital anomalies among young infants admitted at Bugando medical centre, Mwanza, Tanzania
}

\author{
Florentina Mashuda ${ }^{1}$, Antke Zuechner ${ }^{2}$, Phillipo L Chalya ${ }^{3}$, Benson R Kidenya ${ }^{4}$ and Mange Manyama ${ }^{5^{*}}$
}

\begin{abstract}
Background: Congenital anomalies or birth defects are among the leading causes of infant mortality and morbidity around the world. The impact of congenital anomalies is particularly severe in middle- and low-income countries where health care resources are limited. The prevalence of congenital anomalies varies in different parts of the world, which could reflect different aetiological factors in different geographical regions.
\end{abstract}

Methods: Between October 2012 and January 2013, a cross-sectional study was conducted involving young infants below 2 months of age, admitted at a university teaching hospital in Tanzania. Face-to-face interviews with parents/ caretakers of young infants were carried out to collect socio-demographic and clinical information. Physical examinations were performed on all young infants. Echocardiography, X-ray, cranial as well as abdominal ultrasonographies were performed when indicated.

Results: Analysis of the data showed that among 445 young infants enrolled in the study, the prevalence of congenital anomalies was 29\%, with the Central Nervous System (CNS) as the most commonly affected organ system. Maternal factors that were significantly associated with congenital anomalies included the lack of peri-conceptional use of folic acid $(\mathrm{OR}=3.1 ; 95 \% \mathrm{Cl}=1.4-6.7 ; \mathrm{p}=0.005)$, a maternal age of above 35 years $(\mathrm{OR}=2.2 ; 95 \% \mathrm{Cl}=1.1-4.3$; $\mathrm{p}=0.024)$ and an inadequate attendance to antenatal clinic $(\mathrm{OR}=2.1 ; 95 \% \mathrm{Cl}=1.4-3.3 ; \mathrm{p}<0.001)$. Infant factors that were significantly associated with congenital anomalies were female sex, a birth weight of $2.5 \mathrm{~kg}$ or more, singleton pregnancy and a birth order above 4.

Conclusions: Due to the high prevalence of congenital anomalies observed in this particular context, the hospital should mobilize additional resources for an optimal and timely management of the patients with congenital anomalies. In this study, the proportion of women taking folic acid supplements during early pregnancy was very low. Efforts should be made to ensure that more women use folic acid during the peri-conceptional period, as the use of folic acid supplement has been linked by several authors to a reduced occurrence of some congenital anomalies.

\section{Background}

Congenital anomalies or birth defects are structural or functional anomalies, including metabolic disorders, which are present at the time of birth. Some of the congenital anomalies may be life threatening, may impair function or interfere with the cosmetic value of an individual, hence an immediate management is required. The long-term disability caused by congenital anomalies may have a significant impact not only on a child's well being

\footnotetext{
*Correspondence: manyama73@yahoo.com

${ }^{5}$ Department of Anatomy and Cell Biology, Catholic University of Health and Allied Sciences, Mwanza, Tanzania

Full list of author information is available at the end of the article
}

and development, but also on families, health care systems and societies [1]. The impact of congenital anomalies is severe in middle- and low-income countries. As a matter of fact, it is estimated that approximately $95 \%$ of the children who die from birth defects are from those countries [1].

Worldwide, the incidence of congenital anomalies varies between geographical regions but it is estimated that 3-7\% of children are born with birth defects [1-3]. Approximately, 270,000 newborns die during the first 28 days of life every year from congenital anomalies [4]. In the United States of America, congenital anomalies reportedly affect $2-5 \%$ of all live births [3]. The magnitude of congenital anomalies in Asia has been shown to vary with 
reported incidences of $2.5 \%$ in India and $1.3 \%$ in China $[5,6]$. In the Middle East, where consanguineous marriages are common, the prevalence of major congenital anomalies is reported to be $2-2.5 \%$, the highest prevalence (7\%) being found in consanguineous marriages [1,5,7]. In Africa, some of the rare studies on congenital anomalies have reported an incidence between $1.5 \%$ and 2.5\% in Egypt and East Africa (Kenya and Uganda) respectively [8-10]. Reports on the incidence of congenital anomalies in the developing world should be taken with caution, as the absence of birth defect registries in most of these countries, the deficiency in diagnostic capabilities and unreliable medical records and health statistics might increase the chances of underestimation. Most of the studies done in Africa have been retrospective hospital-based studies which are usually affected by underreporting and other sources of ascertainment bias [11].

About $60 \%$ of the causes of congenital anomalies in humans is still unknown [12]. However, in about $25 \%$ of congenital anomalies, the causes seem to be "multifactorial", indicating a complex interaction between genetic and environmental risk factors [12]. A wide range of environmental risk factors have been associated with the occurrence of congenital anomalies [12]. Exposure during pregnancy to drugs such as thalidomide and phenytoin, alcohol, cigarette smoking, certain environmental chemicals and high doses of radiation have all been implicated in the causation of congenital anomalies [13-15]. The occurrence of congenital anomalies has also been associated with advanced maternal and paternal age, parental consanguinity, increasing birth order and low birth weight $[14,16]$.

The pattern of congenital anomalies varies from region to region and also over time [2]. Generally, congenital anomalies that involve the CNSand the cardiovascular and musculoskeletal systems have been reported to be the most common $[4,12,17]$.

Epidemiological surveys of congenital anomalies in various parts of the world with different environment, socioeconomic status are likely to give out vital information on the prevalence, pattern and risk factors for congenital anomalies in different areas [18]. The current study was conducted in order to determine the prevalence, pattern and factors associated with congenital anomalies among young infants admitted at a university hospital (Bugando Medical Centre), Mwanza, Tanzania. Tanzania is a lowresource African country where the magnitude of congenital anomalies and the associated factors are not well documented. Results from this study will support the development of strategies for improving the management and rehabilitation of patients with congenital anomalies in this particular context. Information on associated factors may shed light on their roles as risk factors for the occurrence of congenital anomalies hence providing baseline data for future studies and public health measures.

\section{Methods}

\section{Study setting and patients recruitment}

This was a cross-sectional hospital-based study involving young infants below 2 months of age admitted at the Bugando Medical Centre between October 2012 and January 2013. The Bugando Medical Centre is a 1000-bed capacity referral hospital in Tanzania serving about 15 million people. The hospital handles most of the congenital anomaly cases of the regions around Lake Victoria because of its capacity to offer specialized management. We enrolled young infants admitted at neonatal wards, pediatrics surgical ward, general pediatrics wards and pediatrics semi-intensive/critical care units. The ethical approval was obtained from the joint Catholic University of Health and Allied Sciences (CUHAS)/BMC ethical review board. The sample size was calculated using the Yamane Taro formula [19], whereby a total of 445 young infants with and without congenital anomalies were recruited in the study. Patients were recruited serially until the desired sample size was reached. After a written consent was obtained from the parents/caretakers, face-to-face interviews with parents/ caretakers of young infants were carried out to collect socio-demographic and clinical information, such as maternal age and parity, history of Diabetes Mellitus, drug intake, exposure to X-ray, history of congenital malformation in the family, parental consanguinity, residential area, maternal exposure to pollutants and number of antenatal clinic visits. The birth weight of young infants was obtained by asking the mother/caretaker and then confirmed by observation of the antenatal card, infants Reproductive and Child Health number one (RCH1) card or other hospital documents, e.g. patient files.

\section{Physical examination and imaging investigations}

All young infants had a thorough physical examination (general and systemic) performed by the paediatrician. Echocardiography, X-ray imaging, cranial and abdominal ultrasonography were performed when required. X-ray films were interpreted by two independent radiologists. Ultrasonography was performed by the radiologists and the senior sonographer. Echocardiography was executed by a paediatrician experienced in paediatric echocardiography. Due to lack of relevant equipment and qualified staff, genetic screening could not be performed.

The patterns of congenital anomalies were classified according to the International Statistical Classification of Diseases and Related Health Problems 10th Revision (ICD-10) Version for congenital malformations, deformations and chromosomal abnormalities [20]. Young infants with multiple congenital anomalies were grouped depending on whether those anomalies qualified as a specific syndrome or not. If they qualified as a specific syndrome, they were then categorized into that syndrome. If no syndrome could be classified by those anomalies and two 
systems were involved, both systems were recorded. When more than two systems were involved, it was recorded as multiple congenital anomalies.

\section{Data management and statistical analysis}

Data were managed using EpiData version 3.1 (Atlanta, US) and analysis was done using STATA version 11 (College Station, Texas). Categorical variables were summarized as proportions and were compared using Pearson's Chi square test while continuous data was described as medians (interquartile range). Univariate followed by multivariate logistic regression analyses were applied to determine the factors associated with congenital anomalies. Factors with a $\mathrm{p}<0.1$ on univariate analysis were subjected to multivariate logistic regression analysis. Crude (unadjusted) and adjusted odds ratios were calculated to quantify the strength of association between the factors and congenital anomalies. The 95\% confidence intervals were determined and the factors with a p-value of less than 0.05 were considered to have a significant association with congenital anomalies.

\section{Results}

Among the 445 young infants admitted at BMC, 243 (54.6\%) were males, 200 (44.9\%) were females and 2 $(0.5 \%)$ had ambiguous genitalia. The age range was between one day and 60 days, with a median age of 4 with an inter-quartile range of 2-8 days. Singleton and twins accounted for $396(89 \%)$ and 49 (11\%) respectively, while a birth order of equal or less than four accounted for 367 (83\%). A family history of congenital anomalies was found among 75 (3\%) infants of the study sample.

Parental demographics showed that 339 (76.7\%) of the mothers of young infants were aged between 20 and 35 years of age and only 67 (15\%) had used folic acid supplements during the first trimester of last pregnancy. Fifty mothers (11\%) were exposed to passive smoking, 12 (3\%) used alcohol during pregnancy and none had a history of active smoking or exposure to X-ray irradiation during pregnancy. There was no history of consanguinity and only one mother was using antiretroviral drugs during her pregnancy.

During the study period, 131 infants among the 445 examined were found with congenital anomalies. This gives a prevalence rate of $29 \%$. The most affected body system was the central nervous system which accounted for 39 of the cases $(29.8 \%)$, followed by the musculoskeletal and gastrointestinal systems for 30 (22.9\%) and 12 (9.2\%) cases respectively (Figure 1). Among the infants with CNS malformations, spina bifida was the most common, followed closely by hydrocephalus, with 16 (38.5\%) and 14 (35.9\%) cases respectively (Table 1). Other CNS congenital anomalies are shown in Table 1.

Omphalocele was found to be the commonest congenital anomaly affecting the musculoskeletal system, accounting for $8(26.8 \%)$ of all cases with musculoskeletal anomalies, followed by gastroschisis $6(20.0 \%)$ and polydactyl 5 (16.7\%) [Table 2]. The most common anomaly of the gastrointestinal tract (GIT) was imperforate anus, accounting for 7 (58\%) of all GIT malformations (Table 2). Other malformations of the musculoskeletal and GIT are shown in Table 2.

Maternal factors that were associated with congenital anomalies were: Non use of folic acid during pregnancy $(\mathrm{OR}=3.1 ; 95 \% \mathrm{CI}=1.4-6.7 ; \mathrm{p}=0.005)$, a maternal age above 35 years $(\mathrm{OR}=2.2 ; 95 \% \mathrm{CI}=1.1-4.3 ; \mathrm{p}=0.024)$, and three or less antenatal clinic (ANC) visits $(\mathrm{OR}=2.1 ; 95 \%$ $\mathrm{CI}=1.4-3.3 ; \mathrm{p}<0.001$ ) [Table 3]. Infant factors that were significantly associated with congenital anomalies were: Female sex $(\mathrm{OR}=1.8 ; 95 \% \mathrm{CI}=1.1-2.8 ; \mathrm{p}=0.013)$, a birth weight of $2.5 \mathrm{~kg}$ or more $(\mathrm{OR}=2.3$; $95 \% \mathrm{CI}=1.4-3.9 ; \mathrm{p}=$ $0.002)$, singleton pregnancy $(\mathrm{OR}=3.5 ; 95 \% \mathrm{CI}=1.2-10.9$; $\mathrm{p}=0.027)$ and a birth order of 4 and above $(\mathrm{OR}=4.4 ; 95 \%$ $\mathrm{CI}=2.6-7.6 ; \mathrm{p}<0.001)$ [Table 4].

\section{Discussion}

Congenital anomalies are among the major causes of childhood morbidity and mortality in many countries around the world. The objective of this cross-sectional study was to report on the prevalence, pattern as well as factors associated with congenital anomalies among young infants admitted at the Bugando Medical Centre in Mwanza, Tanzania. Due to our study design, it was not possible to determine a causal association of these factors with congenital anomalies. In addition, the limited investigative ability at BMC, made it impossible to perform genetic/chromosomal or metabolic disorders studies.

About 29\% of all young infants admitted at BMC during the study period were found to have various congenital anomalies. This prevalence is high compared the data previously reported in developing countries [8-10,21,22]. The high prevalence of congenital anomalies in the present study may be explained by the methodology employed by looking for congenital anomalies among young infants admitted to a tertiary referral hospital. Most young infants with congenital anomalies from regions surrounding Lake Victoria are referred to Bugando Medical Centre, as this is the only referral and consultant hospital capable of performing specialized investigation as well as pediatric surgical care. In addition, the high prevalence observed in this study could also be attributed to the type of classification used in this study (ICD10) which does not differentiate minor and major anomalies. A high prevalence of congenital anomalies of about $13 \%$ has also been reported among neonates admitted in neonatal intensive care units in low income countries [1]. Studies carried out among deliveries in tertiary hospitals have reported lower prevalence ranging between $1 \%$ and $7 \%$ [8-10,21,22]. 


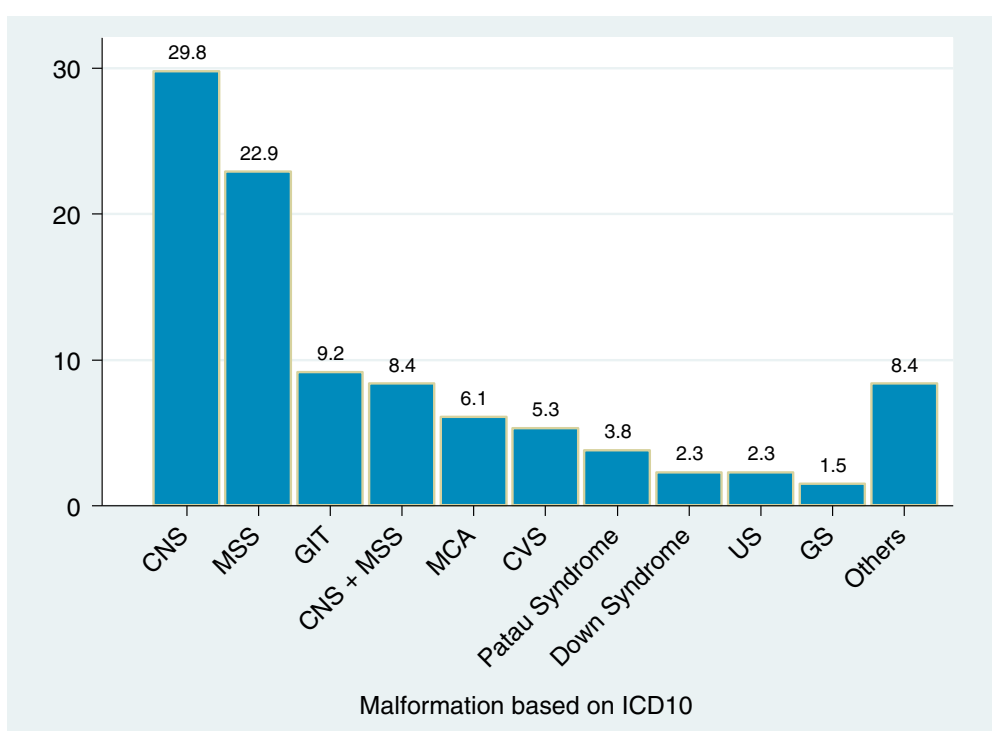

Figure 1 Distribution of congenital anomalies according to ICD 10 among young infants admitted at Bugando Medical Centre from October 2012 to Jan 2013. Key: CNS: central nervous system, MSS: musculoskeletal system, GIT: gastrointestinal tract, MCA: multiple congenital anomalies involving at least 3 systems, CVS: cardiovascular system, US: urinary system, GS: genital system. Others: CNS + GIT, CVS + GIT, GIT + GS, GIT + MSS, MSS + CVS, Pierre Robbin's syndrome, Skin, Skin + MSS.

In the current study, congenital anomalies affecting the CNS were the most common. This could be due to the fact that the surgical management of these malformations needs qualified personnel and special devices, such as ventricular-peritoneal shunt tubes, only available at BMC. Similar studies in Africa have reported a similar trend $[23,24]$. Periconceptual multiple vitamin supplements containing folic acid have been reported to reduce the incidence of neural tube defects and orofacial clefts [25-28]. Our results show that only $15 \%$ of the mothers of young infants had used folic acid during the first trimester of pregnancy and that non use folic acid was significantly associated with congenital anomalies. Due to the study design, the results however, were likely affected by many factors, including recall bias.. Our findings are similar to what was reported in Kenya and India where

Table 1 Specific CNS congenital anomalies among young infants with CNS anomalies

\begin{tabular}{lc}
\hline CNS anomalies & Number (\%) \\
\hline Spina bifida & $16(41.1)$ \\
Spina bifida and hydrocephalus & $1(2.6)$ \\
Abnormal brain tissue & $1(2.6)$ \\
Encephalocele and microcephaly & $5(12.8)$ \\
Hydrocephalus & $14(35.9)$ \\
Hydrocephalus with Dandy Walker cyst & $1(2.6)$ \\
Meningoencephalocele & $1(2.6)$ \\
Total & $\mathbf{3 9 ( 1 0 0 )}$ \\
\hline
\end{tabular}

CNS, musculoskeletal system and gastrointestinal tract were the most affected systems [5,8]. Similar studies elsewhere have reported that the musculoskeletal and gastrointestinal systems are the body systems most commonly affected $[9,18]$. Differences in the pattern of body systems affected by congenital anomalies among different populations could reflect aetiological factors, such as genetic and environmental factors [2].

A significant association between congenital anomalies and the lack or peri-conceptional use of folic acid was found in this study. Folic acid is known to be necessary for the growth and smooth function of human cells, as it is crucial for the biosynthesis and methylation of deoxyribonucleic acid (DNA) and ribonucleic acid (RNA) [25]. This is important for cell division, differentiation and regulation of gene expression, especially at a time of rapid cell division

Table 2 Musculoskeletal and gastrointestinal tract anomalies among young infants

\begin{tabular}{lc}
\hline Musculoskeletal system anomalies & Number (\%) \\
\hline Gastroschisis & $6(20.0)$ \\
Omphalocele & $8(26.7)$ \\
Polydactyly & $5(16.7)$ \\
Talipes & $4(13.3)$ \\
Gastrointestinal tract anomalies & \\
Imperforate anus & $7(58.3)$ \\
Oesophageal atresia & $2(16.7)$ \\
Ankyloglossia (tongue tie) & $2(16.7)$ \\
Cleft lip and palate & $1(8.3)$ \\
\hline
\end{tabular}


Table 3 Univariate and multivariate analysis for parental factors associated with congenital anomalies among young infants admitted at Bugando Medical Centre

\begin{tabular}{|c|c|c|c|c|c|c|}
\hline \multirow[t]{2}{*}{ Parental risk factor } & \multicolumn{2}{|c|}{ Congenital malformations } & \multicolumn{2}{|c|}{ Unadjusted } & \multicolumn{2}{|c|}{ Adjusted } \\
\hline & Yes & No & OR $[95 \% \mathrm{Cl}]$ & $\overline{P \text {-value }}$ & OR [95\% Cl] & P-value \\
\hline \multicolumn{7}{|l|}{ Maternal age } \\
\hline$\leq 35$ & $112(27.6)$ & $293(72.4)$ & 1 & & 1 & \\
\hline$>35$ & $19(47.5)$ & $21(52.5)$ & $2.4[1.2-4.6]$ & 0.010 & $2.2[1.1-4.3]$ & 0.024 \\
\hline \multicolumn{7}{|l|}{ Antenatal visit } \\
\hline$>3$ visit & $51(21.3)$ & $188(78.7)$ & 1 & & 1 & \\
\hline$\leq 3$ visit & 79 (38.9) & $124(61.1)$ & $2.3[1.5-3.6]$ & $<0.001$ & $2.1[1.4-3.3]$ & $<0.001$ \\
\hline \multicolumn{7}{|l|}{ Passive smoking } \\
\hline No & $115(29.3)$ & $277(70.7)$ & 1 & & & \\
\hline Yes & $15(30)$ & $35(70)$ & $1.03[0.5-2]$ & 0.923 & - & - \\
\hline \multicolumn{7}{|l|}{ Maternal alcohol intake } \\
\hline No & $129(30)$ & $301(70)$ & 1 & & & \\
\hline Yes & $1(8.3)$ & $11(91.7)$ & $0.21[0.3-1.7]$ & 0.140 & - & - \\
\hline \multicolumn{7}{|c|}{ Maternal use of folic acid } \\
\hline Yes & $8(11.9)$ & $59(88.11)$ & 1 & & 1 & \\
\hline No & $122(32.5)$ & $253(67.5)$ & $3.6[1.6-7.7]$ & 0.001 & $3.1[1.4-6.7]$ & 0.005 \\
\hline \multicolumn{7}{|l|}{ Paternal age } \\
\hline$<45$ & $117(28.5)$ & $293(71.5)$ & 1 & & & \\
\hline$\geq 45$ & $13(40.6)$ & 19 (59.4) & $1.7[0.8-3.6]$ & 0.152 & _- & _ \\
\hline
\end{tabular}

Table 4 Univariate and multivariate analysis for infant factors associated with congenital anomalies among young infants admitted at Bugando Medical Centre

\begin{tabular}{|c|c|c|c|c|c|c|}
\hline \multirow[t]{2}{*}{ Risk factors } & \multicolumn{2}{|c|}{ Congenital malformations } & \multicolumn{2}{|c|}{ Unadjusted } & \multicolumn{2}{|c|}{ Adjusted } \\
\hline & Yes n (\%) & No n (\%) & OR $[95 \% \mathrm{Cl}]$ & P-value & OR $[95 \% \mathrm{Cl}]$ & P-value \\
\hline \multicolumn{7}{|l|}{ Sex } \\
\hline Male & $64(26.3)$ & $179(73.7)$ & 1 & & 1 & \\
\hline Female & $65(32.5)$ & $135(67.5)$ & $1.3[0.9-2.0]$ & 0.156 & $1.8[1.1-2.8]$ & 0.013 \\
\hline \multicolumn{7}{|l|}{ Birth order } \\
\hline$\leq 4$ & $87(23.7)$ & $280(76.3)$ & 1 & & 1 & \\
\hline$>4$ & $43(57.3)$ & $32(42.7)$ & $4.3[2.6-7.3]$ & $<0.001$ & $4.4[2.6-7.6]$ & $<0.001$ \\
\hline \multicolumn{7}{|c|}{ Pregnancy type } \\
\hline Twin & $4(8.2)$ & $45(91.8)$ & 1 & & 1 & \\
\hline Singleton & $127(32.1)$ & $269(67.9)$ & $5.3[1.9-15.1]$ & 0.002 & $3.5[1.2-10.9]$ & 0.027 \\
\hline \multicolumn{7}{|c|}{ History of birth defect } \\
\hline No & $125(29.1)$ & $304(70.9)$ & 1 & & & \\
\hline Yes & $5(38.5)$ & $8(61.5)$ & $1.5[0.5-4.7]$ & 0.470 & - & - \\
\hline \multicolumn{7}{|l|}{ Birth weight } \\
\hline$<2.5 \mathrm{~kg}$ & $26(16.1)$ & $135(83.9)$ & 1 & & 1 & \\
\hline$\geq 2.5 \mathrm{~kg}$ & $105(37.0)$ & $179(63.0)$ & 3.0 [1.9-4.9] & $<0.001$ & $2.3[1.4-3.9]$ & 0.002 \\
\hline
\end{tabular}


like during embryogenesis [25]. Folic acid is crucial for a normal brain and spinal cord development during the first 4 weeks of gestation [2]. Several studies have shown that folic acid reduces the occurrence of some congenital anomalies e.g. neural tube defects, oro-facial clefts, limb reduction defects, congenital heart defects, urinary system defects and omphalocele [26,27]. The low usage of folic acid during the first trimester of pregnancy in this study could explain the higher proportion of neural tube defects and omphaloceles observed. The association between a low usage of folic acid during pregnancy and the occurrence of congenital anomalies has also been reported elsewhere [28].

The factors found to be significantly associated with congenital anomalies included an inadequate attendance to antenatal clinic, a maternal age above 35 years and a birth order of above 4 children. Antenatal clinic visits are an important part of prenatal care. During those visits, health education is usually given on various issues including adequate nutrition, avoidance of teratogens and maternal infections. Multiple vitamin supplements containing folic acid are also distributed during the clinic sessions [29]. The antenatal visits therefore aim at ensuring a normal pregnancy with the delivery of a healthy baby from a healthy mother. Few $(\leq 3)$ or no prenatal clinic visits have previously been associated with the occurrence of congenital anomalies [30].

Gametogenesis in females begins before birth and the first meiotic division is usually completed shortly before ovulation. Sometimes, the first meiotic division may take a long time to be completed, e.g. up to 45 years. In such circumstances, the chances for meiotic errors from exposure to teratogens are very high because the oocyte spends a longer time in a dividing stage (prophase stage). The risk for congenital anomalies from chromosomal abnormalities as maternal age increases (especially above 35 years) is expected to be high $[8,10,12]$. An association between a high birth order and the occurrence of congenital anomalies was previously reported [2,21]. This link was attributed to the increased rate of mutation after the $3^{\text {rd }}$ gravid compared to the first and second gravid, as well as a higher maternal age [2,31].

A singleton pregnancy, a birth weight of $2.5 \mathrm{~kg}$ and above, a birth order of 4 and above and female sex were also found to be associated with congenital anomalies. We were unable to explain these findings due to the design of our study.

Our findings indicate that maternal smoking (passive smoking), alcohol consumption and a family history of congenital anomalies were not associated with congenital anomalies. Maternal cigarette smoking and alcohol consumption have previously been reported as risk factors for the occurrence of congenital anomalies including orofacial clefts and congenital heart diseases [15,32-34]. Cigarette smoking and alcohol intake are not common among Tanzanian women due to cultural norms. In addition, under ascertainment bias could have also affected our results due to non-reporting of family history because of shame, etc. A family history of birth defects has been associated with an increased risk of having another children with congenital anomalies, with a recurrence rate ranging between 2 and $5 \%$ and 1\% for neural tube defects and Down syndrome respectively [35].

The significance of the association between congenital anomalies and various factors in this study should be interpreted with care. As a matter of fact, the information on associated factors was obtained through interviews and is likely to be affected by recall bias of former exposure to risk variables as well as denial from parents regarding the exposure to some of the factors due to fact occurrence of congenital anomalies is a delicate issue to most families. In addition, our results of associated factors are also likely to be influenced by under-ascertainment and use of BMC as the only data source.

\section{Conclusion}

The high prevalence of congenital anomalies observed in this study calls for a special attention on this problem. We recommend that the hospital should mobilize more resources for an optimal and timely management of patients with congenital anomalies. Large community-based studies should be conducted in Tanzania to determine the prevalence of congenital anomalies among the newborns and their associated factors. The results could shed light on the pattern as well as the various risk factors for congenital anomalies.

In this study, the proportion of women taking folic acid supplements during early pregnancy was very low. Even though, due to its design, our study could not establish a causal relationship between non-use of folic acid and the occurrence of congenital anomalies, efforts should be made to ensure that more women use folic acid during the periconceptional period since there is ample documentation about its association with congenital anomalies Similarly, pregnancies and deliveries at an advanced age should be discouraged as they have been associated in various studies with the occurrence of congenital anomalies. Large community-based studies in different geographical, environmental and socio-economic settings should be conducted in Tanzania to determine the prevalence of congenital anomalies and their associated factors.

\section{Competing interests}

There were no financial or non-financial competing interests.

\section{Authors' contributions}

FM participated in the design of the study, data collection and analysis, as well as helped to draft the manuscript; AZ participated in the design of the study and performed some of the radiological investigations on young infants; PC participated in the design and helped draft the manuscript; BRK participated in statistical analysis and helped draft the manuscript; MM participated in the design of the study, data analysis and drafted the manuscript. All authors read and approved the final manuscript. 


\section{Acknowledgements}

We would like to express our gratitude to the staff of Bugando Medical Centre (departments of Paediatrics, Surgery and Radiology) for the help they provided during the process of data collection. We would also like to thank Dr Godfrey Kasanga for performing some of the radiological investigations on young infants. We appreciate the efforts of the Bugando University College of Health Sciences in providing the funds that enabled us to conduct this study.

\section{Author details}

${ }^{1}$ Department of Pediatrics and Child Health, Catholic University of Health and Allied Sciences, Mwanza, Tanzania. ${ }^{2}$ Department of Pediatrics and Child Health, Bugando Medical Centre, Mwanza, Tanzania. ${ }^{3}$ Department of Surgery, Bugando Medical Centre, Mwanza, Tanzania. ${ }^{4}$ Department of Biochemistry and Molecular Biology, Catholic University of Health and Allied Sciences, Mwanza, Tanzania. ${ }^{5}$ Department of Anatomy and Cell Biology, Catholic University of Health and Allied Sciences, Mwanza, Tanzania.

Received: 15 December 2013 Accepted: 21 March 2014

Published: 29 March 2014

\section{References}

1. Howson CP, Christianson AC, Modell B: Controlling birth defects: reducing the hidden toll of dying and disabled children in low-income countries. Dis Control Prior Proj 2008. http://www.dcp2.org/file/230/dcpptwpcongenitaldefects web.pdf

2. Singh A, Gupta RK: Pattern of congenital anomalies in newborn: a hospital based prospective study. JK Sci 2009, 1:34-36.

3. Sekhobo JP, Druschel CM: An evaluation of congenital malformations surveillance in New York State: an application of centers for disease control and prevention (CDC) guidelines for evaluating surveillance systems. Public Health Rep 2001, 116:296-305.

4. World Health Statistics 2008. Geneva: World Health Organization; 2008

5. Parmar A, Rathod SP, Patel SV, Patel SM: A study of congenital anomalies in newborn. NIJRM 2010, 1:13-7.

6. Emanuel I, Huang SW, Gutman LT, Yu FC, Lin CC: The incidence of congenital malformations in a Chinese population: the Taipe collaborative study. Teratology 1972, 5:159-169.

7. Malla BK: One year review study of congenital anatomical malformation at birth in Maternity Hospital (Prasutigriha), Thapathali, Kathmandu. Kathmandu Univ Med J 2007, 5(4):557-560.

8. Muga R, Mumah S, Juma P: Congenital malformations among newborns in Kenya. Afr J Food Nutr Sci 2009, 9(3):814-829.

9. Ndibazza J, Lule S, Nampijja M, Mpairwe H, Oduru G, Kiggundu M, Akello M, Muhangi L, Elliott AM: Brief report a description of congenital anomalies among infants in Entebbe, Uganda. Clin Mol Teratol 2011, 91:857-861.

10. Shawky RM, Sadik DI: Congenital malformations prevalent among Egyptian children and associated risk factors. Egypt J Med Human Genet 2011, 12(1):69-78

11. Manyama M, Rolian C, Gilyoma J, Magori CC, Mjema K, Mazyala E, Kimwaga E, Hallgrimsson: An assessment of orofacial clefts in Tanzania. BMC Oral Health 2011, 11:5.

12. Moore KL, Persaud TVN: Human birth defects. In The Developing Human: Clinically Orientated Embryology. Philadelphia: Saunders; 2003:157-186.

13. Queißer-Luft A, Spranger J: Congenital malformations. Dtsch Arzteb/ 2006, 103(38):A 2464-A 2471

14. Rittler M, López-Camelo J, Castilla EE: Sex ratio and associated risk factors for 50 congenital anomaly types: clues for causal heterogeneity. Birth Defects Res A Clin Mol Teratol 2004, 70(1):13-19.

15. Little J, Cardy A, Arslan MT, Gilmour M, Mossey PA: Smoking and orofacial clefts: a United Kingdom-based case-control study. Cleft Palate Craniofac J 2004, 41(4):381-386.

16. Taksande A, Vilhekar $K$, Chaturvedi $P$, Jain M: Congenital malformations at birth in Central India: A rural medical college hospital based data. Indian J Hum Genet 2010, 16(3):159-163.

17. Mohamed A, El Koumi, Ehab A, Al Banna, Ibrahim Lebda: Pattern of congenital anomalies in newborn: a hospital-based study. Pediatr Rep 2013, 5:20-23.

18. Ekwere EO, Mcneil R, Agim B, Jeminiwa B: A retrospective study of congenital anomalies presented at a tertiary health facility in Jos, Nigeria. JPCS 2011, 3(3):24-28.
19. Yamane T: Statistics: An Introductory Analysis. New York: Harper \& Row; 1973.

20. International statistical classification of diseases and related health problems 10th revisions. http://apps.who.int/classifications/icd10/browse/2010/en.

21. Swain S, Agrawal A, Bhatia BD: Congenital malformations at birth. Indian Pediatr 1994, 31(10):1187-1191.

22. Mosayebi Z, Movahedian AH: Pattern of congenital malformations in consanguineous versus nonconsanguineous marriages in Kashan, Islamic Republic of Iran. East Mediterr Health J 2007, 13(4):868-875.

23. Delport SD, Christianson AL, van den Berg HJ, Wolmarans L, Gericke GS: Congenital anomalies in black South African liveborn neonates at an urban academic hospital. S Afr Med J 1995, 85(1):11-15.

24. Adeleye AO, Olowookere KG: Central nervous system congenital anomalies: a prospective neurosurgical observational study from Nigeria. Congenit Anom (Kyoto) 2009, 49(4):258-261.

25. Wisniewska K, Wysocki J: The importance of folic acid in the primary prevention of congenital malformations. Arch Perinat Med 2008, 14(2):32-40.

26. Godwin KA, Sibbald B, Bedard T, Kuzeljevic B, Lowry RB, Arbour L: Changes in frequencies of select congenital anomalies since the onset of folic acid fortification in a Canadian birth defect registry. Canad J Public Health 2008, 99(4):271-275.

27. Salerno P: Folic acid in congenital malformations prevention. Ann lg 2009, 22(4):10-12

28. Verity $\mathrm{C}$, Firth $\mathrm{H}$, Constant $\mathrm{C}$ : Congenital abnormalities of the central nervous system. Neurol Neurosurg Psychiatry 2003, 74:i3-i8.

29. Penchaszadeh VB: Preventing congenital anomalies in developing countries. Community Genet 2002, 5:61-69.

30. Granado S: Congenital malformations in Rio de Janeiro, Brazil: prevalence and associated factors. Cad Saude Publica 2006, 22(11):2423-2431.

31. Alverson CJ, Strickland MJ, Gilboa SM, Correa A: Maternal smoking and congenital heart defects in the Baltimore-Washington Infant Study. Official J Am Acad Pediatr 2011, 127(3):647-653.

32. Hazem SEM, Hassan SK, Alaa Eldeen MI, Rami M: Epidemiology of birth defects in Women's health university center Assiut-Egypt: an observational cross-sectional study. Am J Sci 2012, 8:777-781.

33. Hackshaw A, Rodeck C, Boniface S: Maternal smoking in pregnancy and birth defects. Hum Reprod Update 2011, 17(5):589-604.

34. Mehrabi Kushki BZ A: The effect of consanguineous marriages on congenital malformation. J Res Med Sci 2005, 10(5):298-301.

35. El Koumi MA, Al Banna EA, Lebda I: Pattern of congenital anomalies in newborn: a hospital-based study. Pediatr Rep 2013, 5(1):0-3.

\section{doi:10.1186/1756-0500-7-195}

Cite this article as: Mashuda et al:: Pattern and factors associated with congenital anomalies among young infants admitted at Bugando medical centre, Mwanza, Tanzania. BMC Research Notes 2014 7:195.

\section{Submit your next manuscript to BioMed Central and take full advantage of:}

- Convenient online submission

- Thorough peer review

- No space constraints or color figure charges

- Immediate publication on acceptance

- Inclusion in PubMed, CAS, Scopus and Google Scholar

- Research which is freely available for redistribution 